\section{Retinal thickness change in relation to visual acuity improvement after 23-gauge vitrectomy for idiopathic epimacular membrane}

Beyoğlu Eye Training and Research Hospital, Istanbul, Turkey

Correspondence: O Çekiç, Vakif Gureba Training and Research Hospital, Department of Ophthalmology, Aksaray Vatan Caddesi, Fatih, Istanbul 34093, Turkey Tel: + 905326928720 . E-mail: ocekic@hotmail.com

Received: 17 March 2010 Accepted in revised form: 7 October 2010; Published online: 26 November 2010

This paper was presented as a poster at 9th EURETINA Congress, Nice, France, 2009

\begin{abstract}
Purpose To assess the course of macular thickness change in relation to visual acuity improvement, in patients being operated for idiopathic epimacular membrane by 23-gauge pars plana vitrectomy.

Materials and methods The clinical charts of 21 eyes of 19 consecutive patients $(9$ male and 10 female) were assessed retrospectively. Postoperatively, patients were followed up by best-corrected Snellen visual acuity (BCVA), a thorough ophthalmological examination, including fundoscopy, retinal thickness, and macular morphology as demonstrated by optical coherence tomography. The main outcome measures of the study were changed in central macular thickness (CMT)and visual acuity during the follow-up period.

Results The mean duration of follow-up was $15.9 \pm 1.0$ months (range 12-24 months). Mean baseline BCVA improved from $0.22 \pm 0.03$ (decimal fraction) to $0.50 \pm 0.07$ at the last visit $(P=0.001)$, whereas baseline CMT $(408 \mu \mathrm{m})$ decreased to $283 \mu \mathrm{m}$ at final examination $(P=0.001)$. During the follow-up, 10 eyes $(47 \%)$ achieved 0.5 or better BCVA. The first significantly lower CMT readings were obtained at third-month visit while BCVA showed an improvement with the significant difference following 9 months after the surgery. In total, 8 out of $\mathbf{1 1}$ phakic eyes underwent cataract extraction with a mean of 6.8 months (range 1-12 months). Conclusions After a successful epimacular membrane removal, functional recovery takes at least 9 months in comparison to much early
\end{abstract}

resolution in macular oedema that was evident at third month following the surgery. Eye (2011) 25, 180-184; doi:10.1038/eye.2010.176; published online 26 November 2010

Keywords: central macular thickness; idiopathic epiretinal membrane; optical coherence tomography; pars plana vitrectomy; visual acuity

\section{Introduction}

Idiopathic epimacular membrane is one of the macular diseases of good functional recovery potential following vitroretinal surgery. The membrane results from fibroglial proliferation on the inner surface of the retina presumably secondary to a break in internal limiting membrane occurring during the process of posterior vitreous detachment. ${ }^{1}$ The formation of the membrane depends on expression of specific growth factors and cytokines. ${ }^{2}$ Surgical removal of the membrane in patients with significant symptoms improves visual acuity and reduces metamorphopsia in most of the cases, whereas a small number of patients do not benefit from the procedure. Unlike rapid hole closure and visual acuity improvement after vitrectomy in patients with macular holes, eyes with idiopathic epimacular membrane experience later structural and functional recovery. The improvement in visual acuity continues for more than 6 months. ${ }^{3,4}$

The aim of the current study was to assess the course of macular thickness change in relation to visual acuity improvement in 
patients being operated for idiopathic epimacular membrane by 23 -gauge pars plana vitrectomy technique.

\section{Patients and methods}

The clinical charts of 21 eyes of 19 consecutive patients (9 male and 10 female) having been operated for idiopathic epimacular membrane at our clinic between March 2006 and March 2008 were assessed retrospectively. All subjects signed a written informed consent. The diagnosis of epimacular membrane was based on ophthalmoscopy and optical coherence tomography (OCT). Besides preoperative OCT features, and also preoperative best-corrected Snellen visual acuity (BCVA), ophthalmoscopic examination, grade of the membrane, and the demographic features of the patients were noted. Postoperatively, patients were followed-up with BCVA, a thorough ophthalmological examination, including fundoscopy, and retinal thickness and macular morphology by OCT. Patients were carefully examined for cataract progression and retinal or optic atrophy.

General anaesthesia was used in five and subtenon anaesthesia in 16 of the cases. All eyes underwent a standard three-port transconjunctival pars plana vitrectomy using 23-gauge instrumentation. If not present, intentional posterior vitreous detachment was created initially, and the membrane was peeled beginning from an edge by a microforcep. Thereafter, internal limiting membrane was peeled in a circular manner, either by using membrane blue dye (four eyes) or by a triamcinolone-assisted procedure (17 eyes): either adjunctive, for internal limiting membrane staining, was chosen randomly. Triamcinolone, as membrane blue dye, enhances visualization of internal limiting membrane, and the peeled area was seen as an area lacking white specks. ${ }^{5,6}$ All eyes were infused with balanced salt solution-BSS as internal tamponade at the conclusion of surgery. All intraoperative complications were noted. Four eyes underwent combined surgery of vitrectomy and phocoemulsification with intraocular lens implantation procedure.

OCT (Stratus OCT III, Zeiss-Humphrey, Dublin, CA, USA) was used to measure retinal thickness and show retinal and membrane features. Central macular thickness (CMT) was defined as the central 1-mm zone as visualized by fast macular thickness measuring the thickness at six different planes.

The main outcome measures of the study were CMT change in relation to visual acuity improvement during the follow-up period. Total recovery of foveal depression and cystoid macular oedema, being persisted or recurred, were noted. The functional success of the surgery was evaluated by the number of eyes with two or more lines of visual acuity improvement.

All data were collected and assessed by SPSS (SPSS Inc., Chicago, IL, USA) version 11.5 for Windows. For statistical analysis Snellen visual acuity values were converted to equivalent logarithmic Minimum Angle of Resolution values. The change in BCVA and CMT was assessed by non-parametric Willcoxon test. Fisher's exact test was performed to show the relations of visual acuity and baseline macular features and final foveal depression as shown by OCT. Spearman's correlation coefficient was estimated for final BCVA and baseline BCVA, change in BCVA, baseline and final CMT and change in CMT. The $P$-value $<0.05$ was regarded as statistically significant.

We certify that all applicable institutional and governmental regulations concerning the ethical use of human volunteers were followed during this research.

\section{Results}

The mean age was $66.4 \pm 1.6$ (mean \pm SEM) years (range, 51-82 years) and duration of follow-up was $15.9 \pm 1.0$ months (range, 12-24 months).

Mean baseline Snellen BCVA improved from $0.22 \pm 0.03(0.01-0.60)$ to $0.50 \pm 0.07(0.01-1.0)$ at the last visit $(P=0.001)$, whereas baseline mean CMT $(408 \mu \mathrm{m})$ decreased to $283 \mu \mathrm{m}$ at final examination $(P=0.001)$. The first significantly lower CMT readings were obtained at third-month visit, whereas BCVA showed significant improvement as early as 9 months after the surgery (Table 1).

In subgroup analysis, pseudophakic eyes $(n=10$; preoperatively pseudophakic $(n=6)$ and pseudophakic at the conclusion of combined surgery $(n=4))$ showed earliest visual acuity improvement at ninth month $(P=0.045)$. Phakic study eyes $(n=11)$ tended to improve visual acuity at ninth month, but insignificantly $(P=0.06)$. The latter group showed clear significance at twelfth month $(P=0.02)$ compared with baseline

Table 1 Pre- and postoperative mean ( \pm SEM) central macular thickness $(\mu \mathrm{m})$ and BCVA values

\begin{tabular}{lclcl}
\hline Time & CMT & P-value & BCVA & P-value \\
\hline Baseline & $408 \pm 29$ & & $0.22 \pm 0.03$ & \\
First month & $362 \pm 25$ & 0.19 & $0.23 \pm 0.04$ & 0.52 \\
Third month & $309 \pm 21$ & $0.01^{*}$ & $0.27 \pm 0.04$ & 0.55 \\
Sixth month & $306 \pm 25$ & $0.003^{*}$ & $0.36 \pm 0.06$ & 0.06 \\
Ninth month & $312 \pm 26$ & $0.03^{*}$ & $0.49 \pm 0.07$ & $0.006^{*}$ \\
Twelfth month & $259 \pm 24$ & $0.003^{*}$ & $0.52 \pm 0.08$ & $0.003^{*}$ \\
Eighteenth month & $289 \pm 26$ & $0.01^{*}$ & $0.64 \pm 0.08$ & $0.005^{*}$ \\
\hline
\end{tabular}

Abbreviations: CMT, central macular thickness; BCVA, best-corrected visual acuity.

*Indicates significant difference from baseline (Wilcoxon test). 


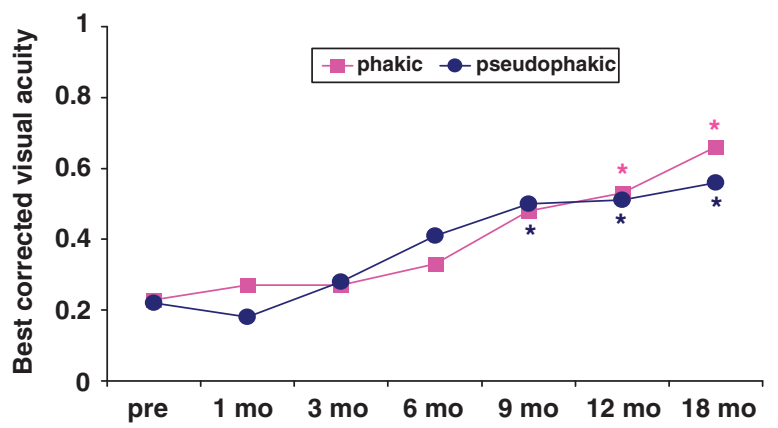

Figure 1 Change in Snellen best-corrected visual acuity in phakic and pseudophakic eyes. Pseudophakic eyes $(n=10$; preoperatively pseudophakic $(n=6)$ and pseudophakic at the conclusion of combined surgery $(n=4))$ showed earliest visual acuity improvement at ninth month $(P=0.045)$. Phakic study eyes $(n=11)$ tended to improve visual acuity at ninth month, but insignificantly $(P=0.06)$. The latter group showed clear significance at twelfth month $(P=0.02)$ compared with baseline. Cataract progression was observed in 10 of 11 phakic eyes during the study period. In total, 8 out of 11 phakic eyes underwent cataract extraction between postoperative 1-12 months *Indicates significance $(P<0.05)$ from baseline evaluated by Wilcoxon test.

(Figure 1). Cataract progression was observed in 10 of 11 phakic eyes. In total, 8 out of 11 phakic eyes underwent cataract extraction with a mean of 6.8 months (range 1-12 months). The other two eyes have been scheduled for cataract extraction.

At final examination, visual acuity improved at least two Snellen lines in $72 \%$ of the eyes while rest of the eyes showed no improvement $(28 \%)$. None of the eyes lost more than one Snellen line of BCVA. In total, 10 eyes (47\%) achieved 0.5 or better BCVA.

At baseline, the membrane was diffusely attached to retina in nine (42\%) eyes, and focally attached in 12 (58\%) eyes. All except one eye had grade 2 epiretinal membranes. Considering the baseline macular features, besides epimacular membrane three eyes had cystoid macular oedema (14\%), four eyes had lamellar hole (19\%), one eye had pseudohole (5\%), four eyes had macular detachment (19\%), and two eyes had vitreomacular traction (9\%). None of the eyes had normal foveal depression before operation.

During follow-up period, the normal foveal depression was achieved in nine (42\%) out of 21 eyes. Cystoid macular oedema disappeared only in one out of three eyes during the follow-up, and another one developed small intraretinal cystic spaces in the macula. Two eyes had extrafoveal residual membrane and one eye had a recurrence of the membrane that required a second operation for epimacular membrane removal 12 months postoperatively.

Comparing the two groups of eyes with improved and unchanged final visual acuity, we could not find any significant difference between OCT features of preoperative macular membranes $(P>0.05)$. In the patients with final BCVA of 0.5 or better, although statistically insignificant, no eye had cystoid macular oedema preoperatively $(P=0.21)$. The normal foveal depression was obtained in 7 out of 10 eyes with 0.5 or better final visual acuity $(P=0.03)$.

The only significant correlation was present between the baseline BCVA and final BCVA (rho $=0.71, P<0.001$ ).

\section{Discussion}

In the current study with eyes that underwent vitrectomy for epiretinal membrane, despite the earlier regression of the macular oedema, improvement in visual acuity took longer time. The first significantly lower CMT readings were obtained at third-month visit, whereas BCVA showed a significant improvement at postoperative 9 months.

As we included phakic and pseudophakic patients together in the present study, and the subject of visual acuity increase after epiretinal membrane surgery might be dependent on the phakic status of eye, we performed a subgroup analysis within the study group to decide whether increase in lenticular opacity was the main determinant for visual acuity prognosis. Pseudophakic eyes showed earliest visual acuity improvement at ninth month $(P=0.045)$. Phakic group tended to improve visual acuity at ninth month, but this improvement was insignificant $(P=0.06)$; visual acuity improvement took 12 months in these eyes $(P=0.02)$. Cataract extraction in our phakic patients was performed at a mean of 6.8 months following vitrectomy. Taking into account the 8 of 12 phakic eyes that underwent cataract extractions till 12 months, it seems that lenticular opacity resulted in 3 months of delay in the recovery of visual acuity after vitrectomy. All results imply that even for pseudophakic eyes, improvement in visual acuity after a successful epimacular membrane removal takes at least 9 months, despite much earlier recovery of CMT.

Delayed rehabilitation of visual acuity compared with anatomic recovery after membrane removal, points out some ultra structural changes possibly related to photoreceptors. The normal functioning of the receptors requires establishment of healthy photoreceptor layer in addition to optimal microenvironment and a good retina pigment epithelial interaction. Experimental studies conducted in eyes of owl monkeys with retinal detachment showed early histopathological changes, such as cystoid spaces in retinal layers, outer segment degeneration and change in shape of retina pigment epithelial cells. ${ }^{7}$ After surgical intervention and repair of retinal anatomy, absorption of the retinal oedema occurred earlier than the recovery of the outer segments 
and retina pigment epithelium morphology that were more protracted. ${ }^{8}$ Likewise the restoration of the vertical arrangement of photoreceptors showed gradual improvement. $^{8,9}$

Photoreceptor disorientation may also have a function in the malfunctioning of the cells. The Stiles-Crawford effect refers to the directional sensitivity of the foveal cone photoreceptors ${ }^{10}$ that in healthy eyes point at the pupil centre. In other words, light hitting the receptors obliquely is less effective at causing stimulation. In pathologies involving retina, for the compromise of normal visual functioning, apart from obvious changes also photoreceptors may show a change in their spatial orientation and become more oblique. Kanis et al reported a decrease in Stiles-Crawford effect function and delayed recovery of Stiles-Crawford effect after 1 year in a case with central serous chorioretinopathy. ${ }^{11}$ In retinal detachment, Stiles-Crawford effect function also showed a progressive improvement over time. ${ }^{12}$

Toxicity of adjunctive dye or desiccation injury from infused air during vitrectomy, as well as retinal microtraumatic effects of the surgery itself might be responsible factors for the anatomic and functional success disparity in a concerted manner.

In this study, three of the six eyes that did not show any visual improvement had had baseline cystoid macular oedema. A previous study showed that eyes with cystoid macular oedema preoperatively had a worse visual outcome after epiretinal membrane removal. ${ }^{4}$ Electron microscopic findings of enucleated eyes with macular oedema due to various retinal pathologies demonstrated the compression of the tissues around the cystic spaces resulting in disorientation and destruction of the photoreceptor cells. ${ }^{13,14}$

A successful epimacular membrane surgery not always results in normal retinal contour and formation of foveal depression. Only a $20 \%$ of foveal depression was reported to recover after removal of the membrane. ${ }^{15}$ In the current study, normal foveal contour was achieved in $42 \%$ of eyes $(n=9)$. Seven of these nine eyes having normal foveal depression recovery demonstrated a final BCVA of 0.5 or better.

Among the BCVA and CMT values (baseline, final and actual change), the only significant correlation was present between the preoperative visual acuity and the final visual acuity. This result is also consistent with previous studies in which the initial visual acuity was found to be one of the most important prognostic factors. $3,16,17$ Likewise, Thompson ${ }^{18}$ reported good final visual acuities in patients operated for epiretinal membrane with good initial visual acuities. The fact that the group of eyes with 0.5 or better final visual acuity had good initial visual acuity and none had cystoid macular oedema preoperatively implies that membrane removal is most beneficial to those eyes that have not already undergone irreversible structural or functional changes in their retina.

The existence of a glial tissue on the functioning retina may lead to abnormal or decreased functioning and loss of normal anatomy of the retina. Because of the tangential traction exerted by the membrane the patient may experience metamorphopsia, vascular leakage, macular oedema, preretinal, and intraretinal haemorrhage and macular detachment. ${ }^{1}$ Also axoplasmic stasis in the nerve fibre layer may occur resulting in decreased visual acuity. ${ }^{1}$ Considering the forementioned functional and anatomical changes, it is important to know progression of the events and recovery of the retina after a successful membrane surgery and to discuss it with the patient especially regarding his or her expectations.

One of the limitations of this study is the use of time domain OCT. With time domain OCT, the findings of cystoid macular oedema are significantly less frequent than with spectral domain. Perhaps spectral domain OCT may show other features related to visual outcome.

In conclusion, after a successful epimacular membrane removal the functional recovery takes at least 9 months in comparison to much earlier resolution in macular oedema that was evident at third month following the surgery. Further controlled studies with larger sample sizes will allow understanding of preoperative determinant features of final visual acuity.

\section{Summary}

\section{What was known before}

- Pars plana vitrectomy provides recovery of anatomic and visual function in eyes with epiretinal membrane.

What this study adds

- Visual acuity improves 6 months later than anatomic recovery following pars plana vitrectomy.

\section{Conflict of interest}

The authors declare no conflict of interest.

\section{References}

1 Johnson TM, Johnson MW. Epiretinal membrane. In: Yanoff M, Duker JS (eds). Ophthalmology, 3rd ed. Elsevier Inc: China, 2004, pp 686-687.

2 Harada C, Mitamura Y, Harada T. The role of cytokines and trophic factors in epiretinal membranes: involvement of signal transduction in glial cells. Prog Retin Eye Res 2006; 25: 149-164.

3 Pesin SR, Olk RJ, Grand MG, Boniuk I, Arribas NP, Thomas MA et al. Vitrectomy for premacular fibroplasia. Prognostic 
factors, long-term follow-up, and time course of visual improvement. Ophthalmology 1991; 98: 1109-1114.

4 Rice TA, De Bustros S, Michels RG, Thompson JT, Debanne SM, Rowland DY. Prognostic factors in vitrectomy for epiretinal membranes of the macula. Ophthalmology 1986; 93: 602-610.

5 Sakamato T, Ishibashi T. Visualizing vitreous in vitrectomy by triamcinolone. Graefes Arch Clin Exp Ophthalmol 2009; 247: 1153-1163.

6 Schadlu R, Tehrani S, Shah GK, Prasad AG. Long-term follow-up results of ilm peeling during vitrectomy surgery for premacular fibrosis. Retina 2008; 28: 853-857.

7 Machemer R. Experimental retinal detachment in the owl monkey. II. Histology of retina and pigment epithelium. Am J Ophthalmol 1968; 66: 396-410.

8 Machemer R. Experimental retinal detachment in the owl monkey. IV. The reattached retina. Am J Ophthalmol 1968; 66: 1075-1091.

9 Anderson DH, Stern WH, Fisher SK, Erickson PA, Borgula GA. Retinal detachment in the cat: the pigment epithelial-photoreceptor interface. Invest Ophthalmol Vis Sci 1983; 24: 906-926.

10 Stiles WS, Crawford BH. The luminous efficiency of rays entering the eye pupil at different points. Proc $R$ Soc Lond B Biol Sci 1933; 112: 428-450.
11 Kanis MJ, van Norren D. Delayed recovery of the optical Stiles-Crawford effect in a case of central serous chorioretinopathy. Br J Ophthalmol 2008; 92: 292-294.

12 Fitzgerald CR, Birch DG, Enoch JM. Functional analysis of vision in patients after retinal detachment repair. Arch Ophthalmol 1980; 98: 1237-1244.

13 Tso MOM. Pathology of cystoid macular edema. Ophthalmology 1982; 89: 902-915.

14 Ripps H, Weale RA. Photo-labile changes and the directional sensitivity of the human fovea. J Physiol 1964; 173: 57-64.

15 Ferreira Saraceno JJ, Mateus IM, Lopes Machado T, Gonçalves VP, Negretto AD, Nakashima Y et al. Study on macular morphology after removal of the idiopathic epiretinal membrane using optical coherence tomography (OCT): a pilot study. Arq Bras Oftalmol 2007; 70: 935-938.

16 Trese MT, Chandler DB, Machemer R. Macular pucker I Prognostic criteria. Graefes Arch Klin Exp Ophthalmol 1983; 221: 12-15.

17 Massin P, Allouch C, Haouchine B, Metge F, Paques M, Tangui L et al. Optical coherence tomography of idiopathic macular epiretinal membranes before and after surgery. Am J Opthalmol 2000; 130: 732-739.

18 Thompson JT. Vitrectomy for epiretinal membranes with good visual acuity. Trans Am Ophthalmol Soc 2004; 102: 97-103. 